\title{
EFFECT OF SURFACE ROUGHNESS ON CHARACTERISTICS OF MAGNETIC FLUID BASED SQUEEZE FILM BETWEEN POROUS CIRCULAR PLATES
}

\author{
P. L. Thakkar *, H. C. Patel \\ Science and Humanities Department, L D College of Engineering, Gujarat, India \\ e-mail: pragnesh83@ldce.ac.in,dr.prof.hcpatel@ldce.ac.in \\ *corresponding author
}

\begin{abstract}
The effect of surface roughness on characteristics of magnetic fluid based squeeze film between porous circular plates is hypothetically analysed. The pressure distribution is obtained by solving concern Reynolds type equation with suitable boundary conditions and the result is utilized to obtain load carrying capacity. It is concluded that the load carrying capacity increases with increasing magnetization, while load carrying capacity decreases due to the standard deviation. It is observed that the negatively skewed roughness and negative mean increase the load carrying capacity. It is also observed that the magnetic fluid lubricant improves the performance of a bearing system, thereby, suggesting that the performance of the bearing with magnetic fluid lubricant is better than the conventional lubricant.
\end{abstract}

Keywords: Magnetic fluid, Squeeze film, Surface roughness, Porosity, Reynolds equation, Pressure distribution, Load carrying capacity

\section{Introduction}

A squeeze film damper is a layer of film between the bearing and the hosing that softens the bearing guide to extend damping effectiveness. Squeeze film behaviour is resolved to show engineering applications in machine tools, gears, bearings, moving components, automotive engines and hydraulic structures and, moreover, in skeletal joints. The squeeze film emerges when one of the lubricating surfaces processes with different ostensible velocity. It takes a specific period for the two surfaces to come in contact due to the pressure developed all through squeeze motion due to viscous resistance to expulsion of the lubricant.

Agrawal (1986) considered a porous inclined slider bearing, lubricated with a magnetic fluid. It was found that the load carrying capacity was greater than that of a viscous porous inclined slider bearing. Bhat and Deheri (1991) analysed theoretically the squeeze film behaviour between two annular discs, when the upper disc with porous facing approached the parallel lower disc. It was concluded that the performance of the bearing with magnetic fluid was better than the conventional lubricant. Prajapati (1995) theoretically analysed the porous squeeze films, lubricated with a magnetic fluid in a bearing of various geometrical shapes like circular, annular, elliptical, infinitely long rectangular, conical, triangular and truncated conical, in the presence of an externally applied oblique magnetic field. Patel et al. (2010) made an investigation of the 
performance of a magnetic fluid based on infinitely long rough bearing with slip velocity. It was concluded that the magnetization may compensate the adverse effect of the standard deviation keeping slip coefficient minimum. Patel et al. (2010) observed the performance of a magnetic fluid based on infinitely short hydrodynamic slider bearing. It was concluded that the magnetic fluid lubricant did not change friction and that load carrying capacity increased as the ratio of the length to outlet film thickness increased.

Deheri et al. (2005) analysed the effect of transverse roughness on the behaviour of slider bearing with squeeze film formed by magnetic fluid. It was noticed that the bearing suffered due to transverse roughness and concluded that the magnetic fluid as a lubricant increased load carrying capacity, decreased the coefficient of friction and affected the centre of pressure marginally. It was also concluded that the effect of magnetization on the plane and secant shaped slider bearings bearing was nominal while the effect on exponential hyperbolic slider bearing was significant. Patel et al. (2012) presented the performance of a hydrodynamic short journal bearing under the presence of magnetic fluid lubricant. It was observed that the load carrying capacity increased nominally while the coefficient of friction decreased significantly and it was also deduced that bearing can support a load even when there is no flow of lubricant. Naduvinamani et al. (2012) conducted a theoretical analysis of the effects of magneto hydrodynamics on the surface roughness on the couple stresses squeeze film lubrication between circular stepped plates. It was observed that the effect of applied magnetic fluid increased the load carrying capacity and delayed the time of approach as compared to corresponding non-magnetic case.

Most of the above studies neglected the effect of surface roughness by considering the bearing surface to be smooth. However, it is known that the bearing surfaces are rough up to a certain extent. Hence, it has been sought to study and analyse the effect of surface roughness on characteristics of magnetic fluid based squeeze film between porous circular plates.

\section{Analysis}

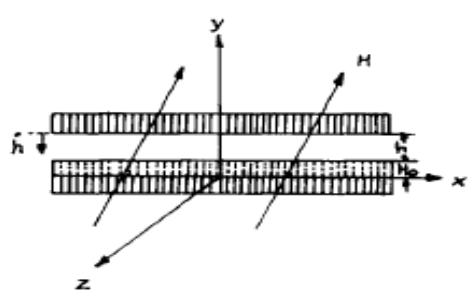

Fig. 1 Configuration of the bearing

Following the method of Christensen and Tonder $(1971,1972,1973)$ the thickness $\mathrm{h}(\mathrm{x})$ is considered as

$$
h(x)=\bar{h}(x)+h_{s}
$$

The related probability density function $f\left(h_{s}\right)$, which is defined by the relationship 


$$
f\left(h_{s}\right)= \begin{cases}\frac{32}{35 b}\left(1-\frac{h_{s}^{2}}{b^{2}}\right)^{3} & -b \leq h_{s} \leq b \\ 0 & \text { elsewhere }\end{cases}
$$

The random variable $h_{s}$ is determined by the relations

$$
\begin{gathered}
\alpha=E\left(h_{s}\right) \\
\sigma^{2}=E\left[\left(h_{s}-\alpha\right)^{2}\right]
\end{gathered}
$$

and

$$
\varepsilon=E\left[\left(h_{s}-\alpha\right)^{3}\right]
$$

where $\mathrm{E}$ denotes the expected value defined by

$$
E(R)=\int_{-c}^{c} R f\left(h_{s}\right) d h_{s}
$$

The details regarding the characterization of the roughness is borrowed from Christensen and Tonder (1971, 1972, 1973). In view of Agrawal (1986) and Bhat and Deheri (1991), the governing modified Reynolds equation for film pressure $\mathrm{P}$, in the case of normal approach of non-rotating porous plates is given by

$$
\frac{\partial^{2} p^{\prime}}{\partial x^{2}}+\frac{\partial^{2} p^{\prime}}{\partial z^{2}}=\frac{12 \mu(d h / d t)}{h^{3}+12 \phi H_{0}}
$$

With $p^{\prime}=p-\frac{1}{2} \mu_{0} \bar{\mu} H^{2}$

With the use of the stochastically averaging method of Christensen and Tonder (1971, 1972, 1973), the equation (7) is transformed to

$$
\frac{\partial^{2} p^{\prime}}{\partial x^{2}}+\frac{\partial^{2} p^{\prime}}{\partial z^{2}}=\frac{12 \mu(d h / d t)}{g(h)+12 \phi H_{0}}
$$

Where $g(h)=h^{3}+3 \sigma^{2} h+3 \alpha^{2} h+3 h^{2} \alpha+3 \sigma^{2} \alpha+\varepsilon+\alpha^{3}+12 \phi H_{0}$

Using the following boundary conditions equation (8) is solved

$$
p(a)=0, \quad\left(\frac{d p}{d r}\right)_{r=0}=0
$$

Where $\frac{x_{1}^{2}}{a^{2}}+\frac{y_{1}^{2}}{b^{2}}=1$

So, the non-dimensional pressure distribution is 


$$
P=-\frac{p h^{3}}{\mu \frac{d h}{d t} a^{2}}=3 \times\left(1-r^{*^{2}}\right) \times\left(\frac{1}{G}+\frac{\mu^{*}}{6}\right)
$$

Where $\sigma^{*}=\frac{\sigma}{h} \quad \alpha^{*}=\frac{\alpha}{h} \quad \varepsilon^{*}=\frac{\varepsilon}{h^{3}} \quad r^{*}=\frac{r}{a} \quad \mu^{*}=-\frac{\mu_{0} \bar{\mu} h^{3}}{\mu \frac{d h}{d t}} \quad \psi=\frac{\phi H_{0}}{h^{3}}$

$$
G=1+3 \sigma^{*^{2}}+3 \alpha *^{2}+3 \alpha *+3 \sigma^{*^{2}} \alpha *+\alpha *^{3}+\varepsilon^{*}+12 \psi
$$

Using pressure distribution, the non-dimensional load carrying capacity of squeeze film

$$
W=-\frac{h^{3} w}{\mu \frac{d h}{d t} \pi a^{4}}=\frac{3}{2} \times\left(\frac{1}{G}+\frac{\mu^{*}}{6}\right)
$$

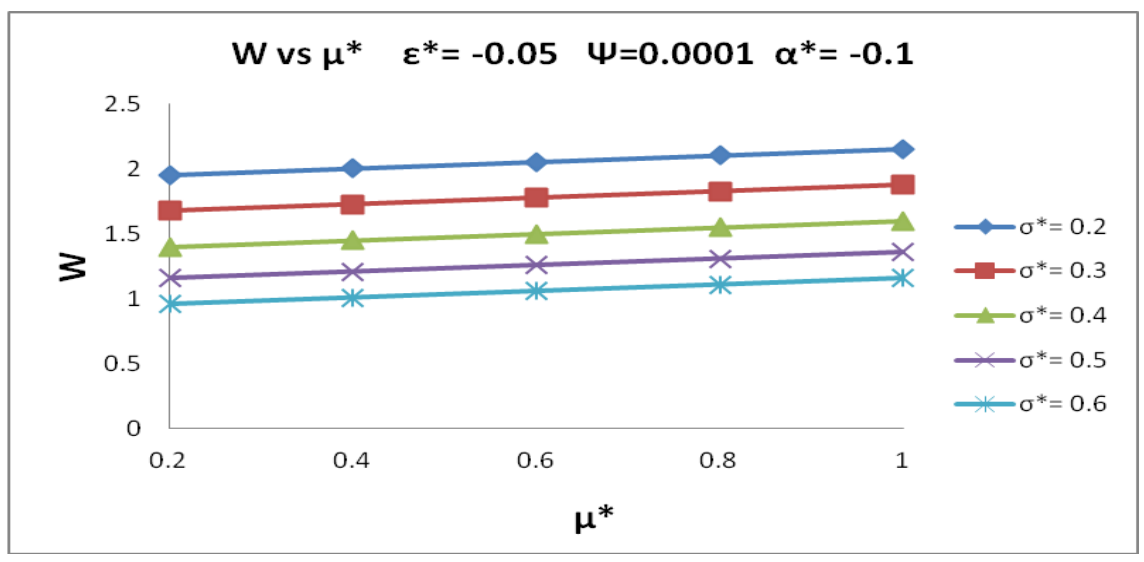

Fig. 2 Non-dimensional load carrying capacity W w.r.t. $\mu^{*}$ for different values of $\sigma^{*}$ 


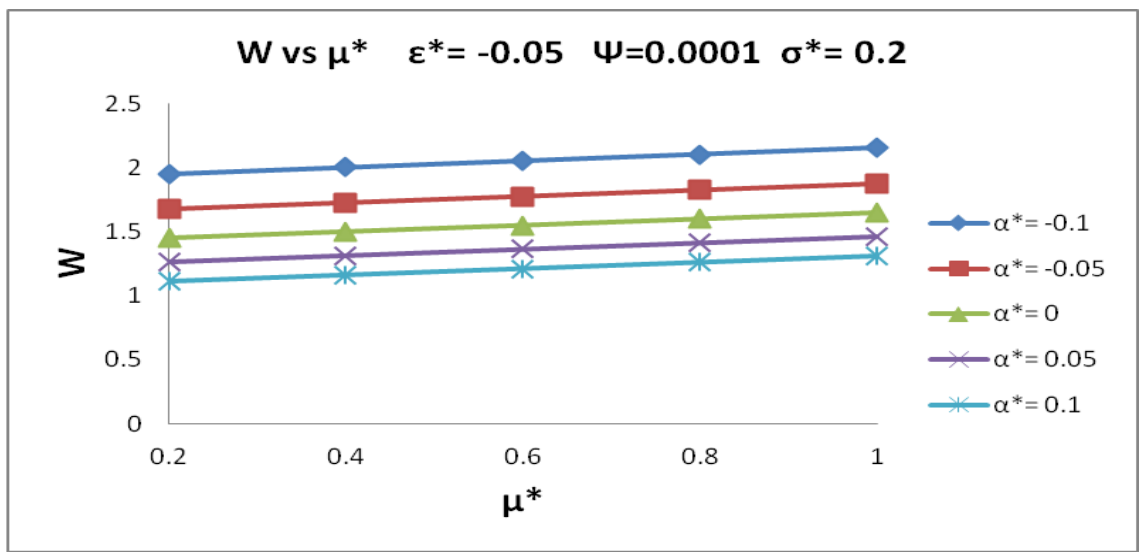

Fig. 3 Non-dimensional load carrying capacity W w.r.t. $\mu^{*}$ for different values of $\alpha^{*}$

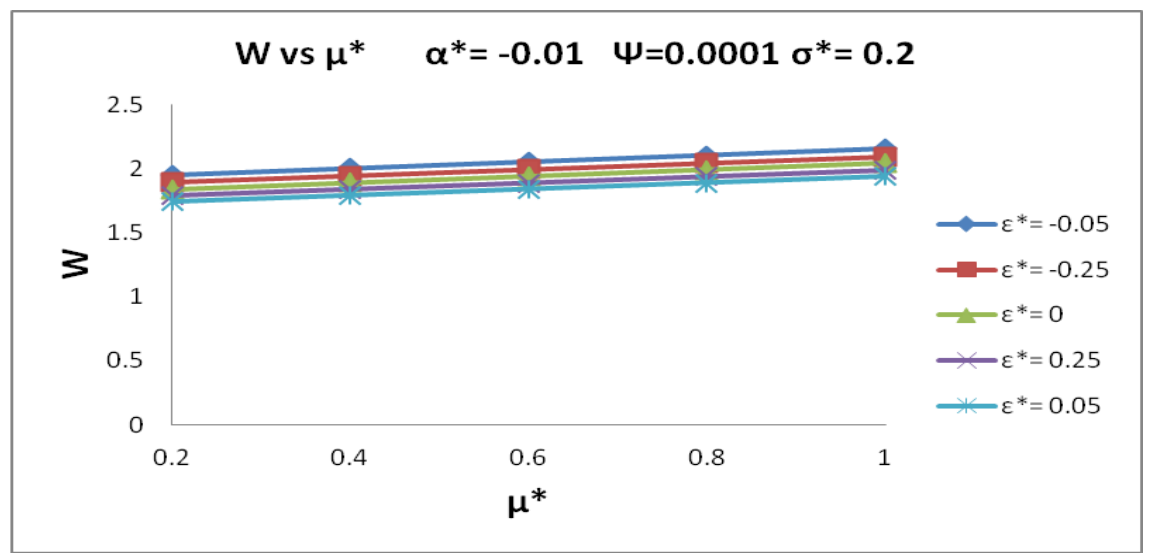

Fig. 4 Non-dimensional load carrying capacity W w.r.t. $\mu^{*}$ for different values of $\varepsilon^{*}$

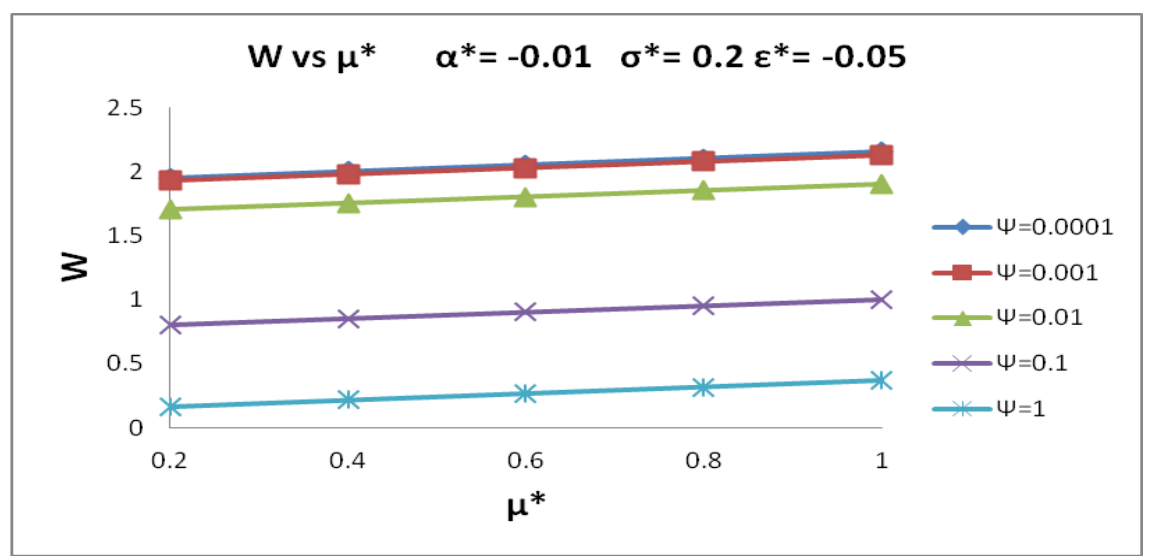

Fig. 5 Non-dimensional load carrying capacity W w.r.t. $\mu^{*}$ for different values of $\Psi$ 


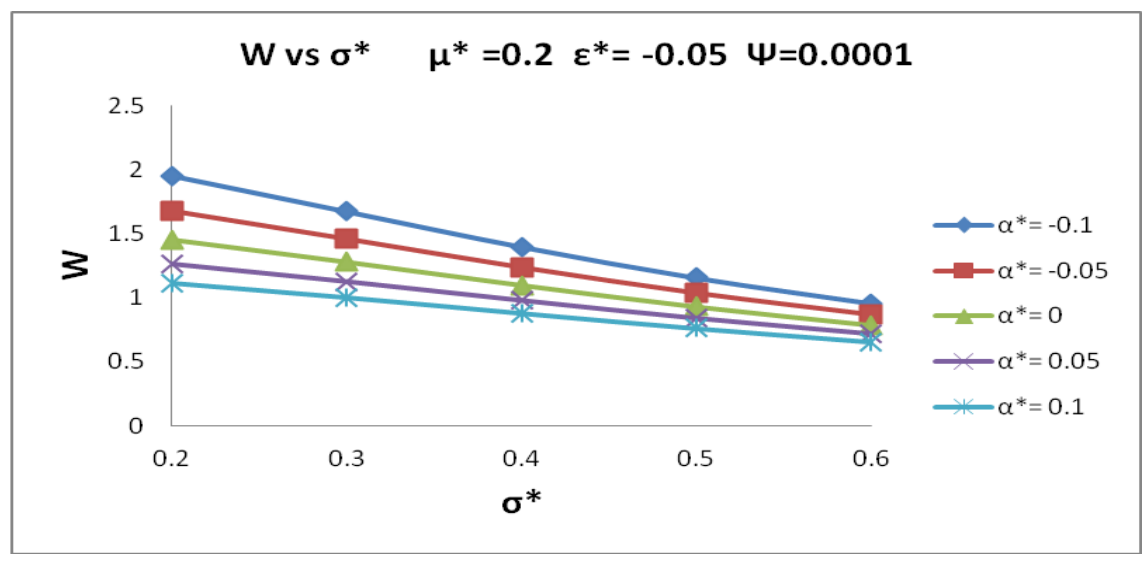

Fig. 6 Non-dimensional load carrying capacity W w.r.t. $\sigma^{*}$ for different values of $\alpha^{*}$

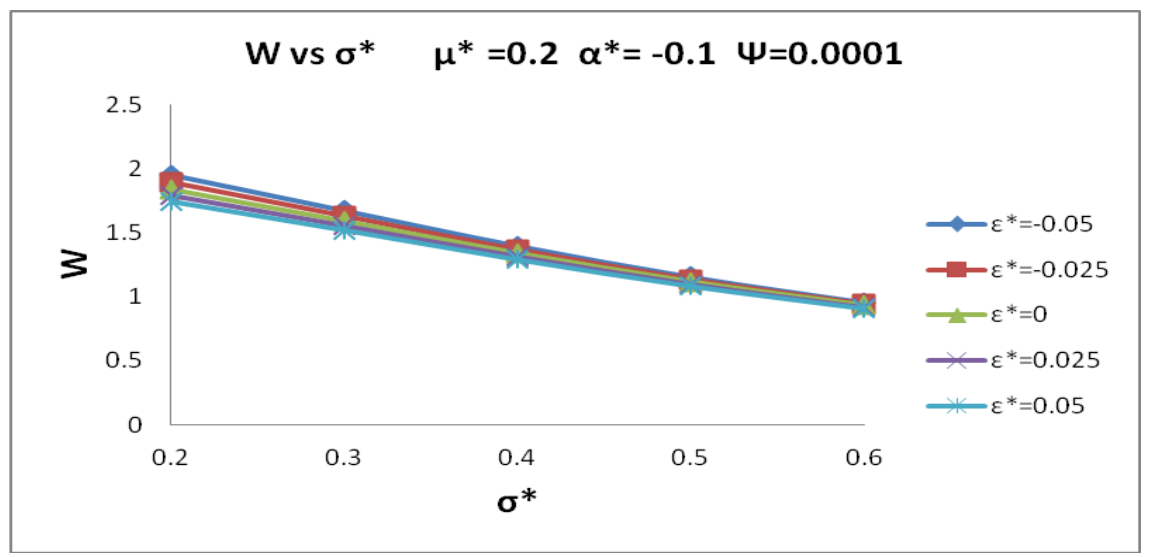

Fig. 7 Non-dimensional load carrying capacity W w.r.t. $\sigma^{*}$ for different values of $\varepsilon^{*}$

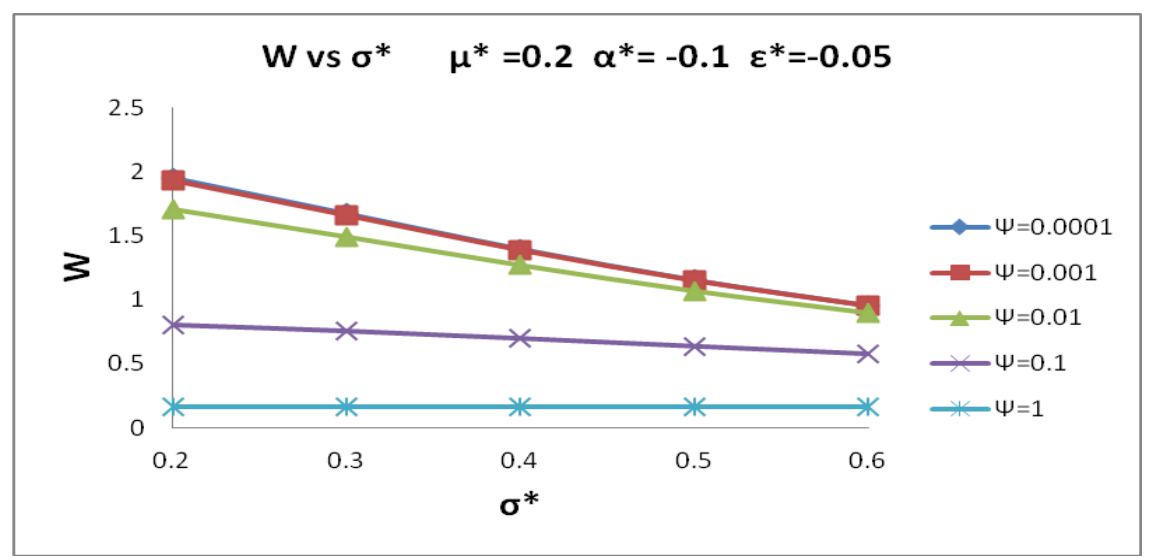

Fig. 8 Non-dimensional load carrying capacity W w.r.t. $\sigma^{*}$ for different values of $\Psi$ 


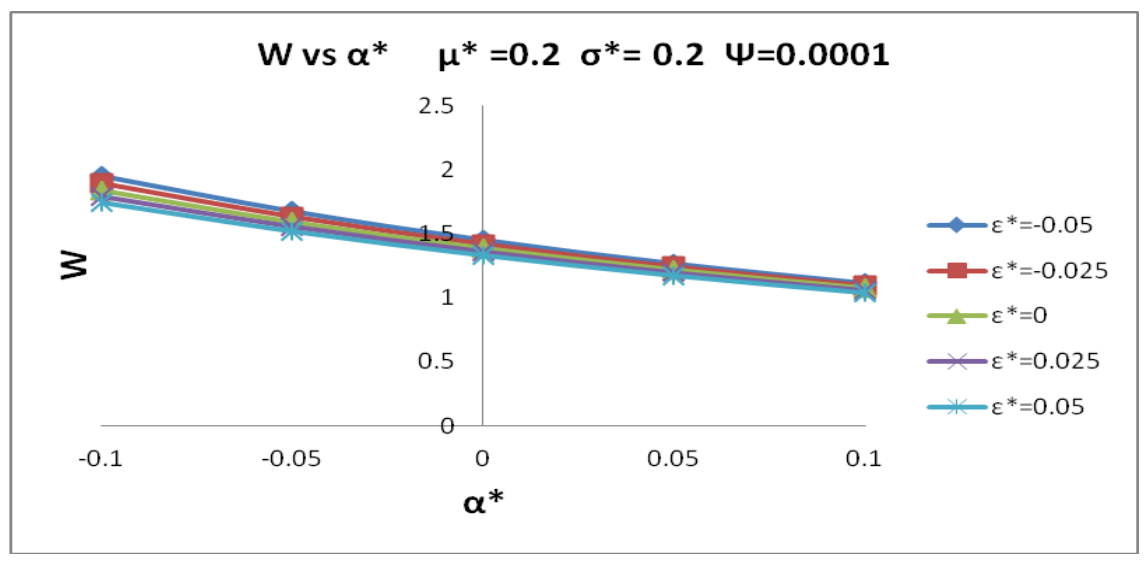

Fig. 9 Non-dimensional load carrying capacity W w.r.t. $\alpha^{*}$ for different values of $\varepsilon^{*}$

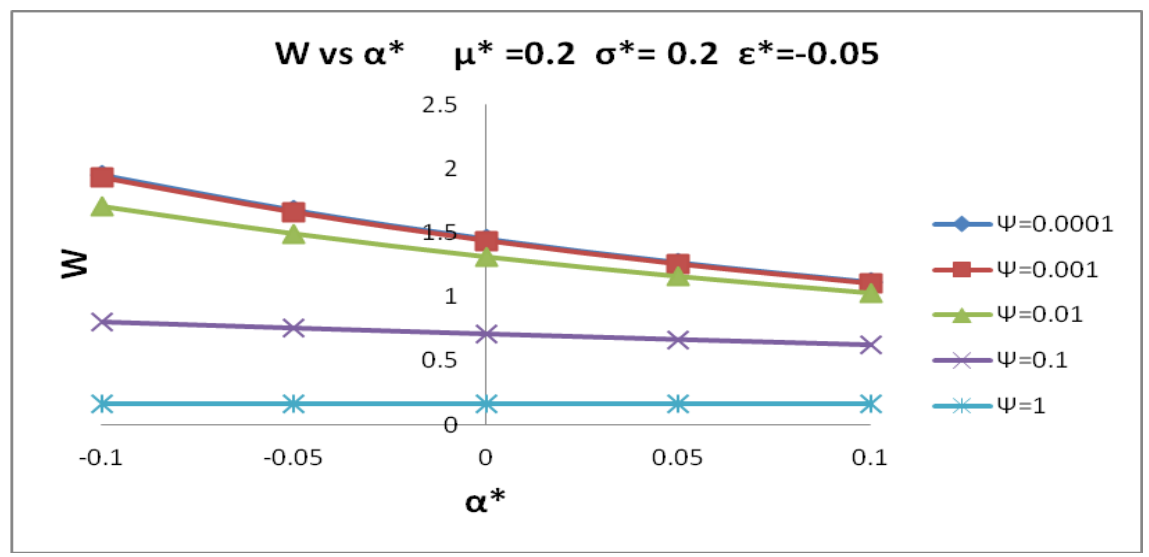

Fig. 10 Non-dimensional load carrying capacity W w.r.t. $\alpha^{*}$ for different values of $\Psi$

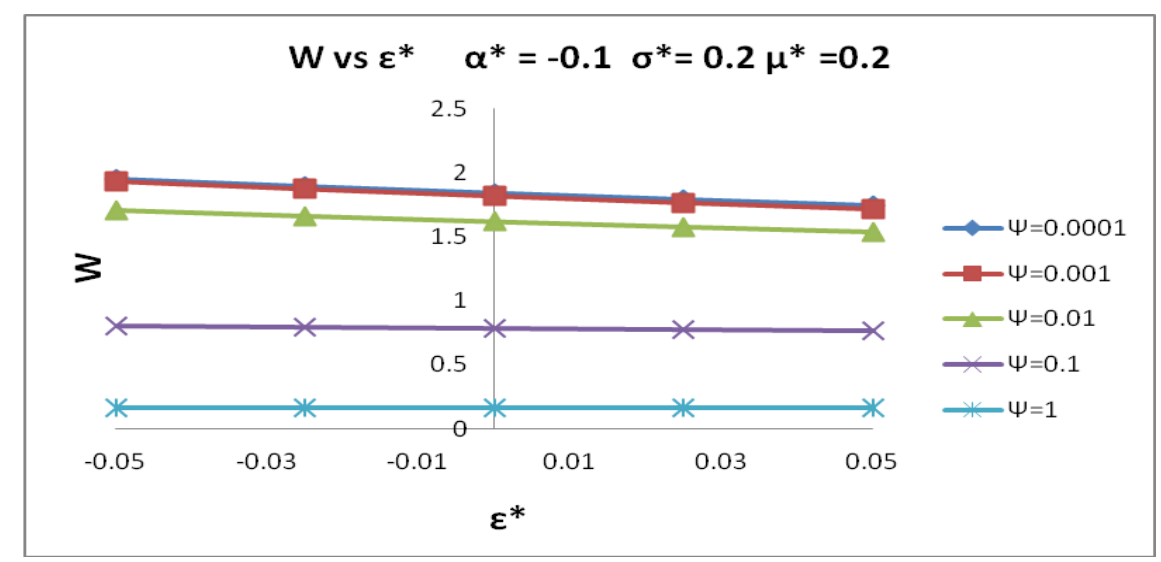

Fig. 11 Non-dimensional load carrying capacity W w.r.t. $\varepsilon^{*}$ for different values of $\Psi$ 


\section{Result and Discussion}

The variation of load carrying capacity with respect to magnetization parameter for various values of standard deviation, mean, skewness and porosity is presented in Figures 2-5. From Figures 2 and 3 , it is observed that the load carrying capacity increases with respect to magnetization parameter which indicates that the performance of the bearing system improves considerably due to the magnetic fluid. From Fig. 4 it can be seen that the effect of skewness on load carrying capacity with respect to magnetization parameter is negligible. Fig. 5 indicates that the values of load carrying capacity with respect to magnetization parameter is high for $\Psi=0.0001$ compared to other values of porosity.

The effect of standard deviation on the distribution on load carrying capacity is demonstrated in Figs. 6-8. From the figures, one can observe that the standard deviation has adverse effects on the performance of bearing system as it decreases the load carrying capacity. The effect of the mean on distribution of load carrying capacity is depicted in Figs. 9-10. It is very clear that the mean (+ve) decreases the load carrying capacity while mean (-ve) increases the load carrying capacity. Figure (11) representing the effect of skewness indicates that it follows the path of mean regarding the trends of load carrying capacity.

\section{Conclusion}

It is revealed that the magnetization has an overall positive effect on the performance of the bearing system. It is also concluded that the adverse effect of the standard deviation and porosity can be minimized by the magnetization parameter in the case of negatively skewed roughness when negative mean occurs. Further, this examination clarifies that compulsory the roughness must be given due idea while designing bearing system even if the suitable value of magnetization parameter has been chosen.

\section{Nomenclature}

$$
\begin{array}{ll}
\alpha: \text { Mean } & \mathrm{H}: \text { External magnetic field } \\
\sigma: \text { Standard deviation } & \mu: \text { Coefficient of viscosity } \\
\varepsilon: \text { Skewness } & \bar{\mu}: \text { The magnetic susceptibility } \\
\alpha^{*}: \text { Non dimensional } & \mu_{0}: \text { Free space magnetic } \\
\text { mean } & \text { permeability } \\
\sigma^{*}: \text { Non dimensional } & \phi: \text { Permeability of porous matrix } \\
\text { standard deviation } & \frac{d h}{d t}: \text { Normal velocity of approach } \\
\varepsilon^{*}: \text { Non dimensional } & \mu^{*}: \text { Magnetization parameter } \\
\text { skewness } & \bar{h}: \text { Mean film thickness } \\
p: \text { Lubricant Pressure } & h_{s}: \text { Deviation from mean film } \\
\text { (N/mm } \left.{ }^{2}\right) & \text { thickness } \\
P: \text { Non dimensional } & H_{0}: \text { Initial film thickness } \\
\text { Pressure } & \\
w: \text { Load carrying } & \\
\text { capacity (N) } & \\
W: \text { Non dimensional } & \\
\text { load carrying capacity } &
\end{array}
$$




\section{References}

Agrawal, V. K (1986). Magnetic-fluid-based porous inclined slider bearing. Wear 107.2,133-139.

Bhat, M. V., and G. M. Deberi (1991). Squeeze film behaviour in porous annular discs lubricated with magnetic fluid. Wear 151.1,123-128.

Christensen, H., and K. Tonder (1971). The hydrodynamic lubrication of rough bearing surfaces of finite width." Journal of Tribology 93(3), 324-329.

Christensen, H., and K. Tonder (1973). The hydrodynamic lubrication of rough journal bearings. Journal of Tribology 95(2),166-172.

Deheri, G. M., P. I. Andharia, and R. M. Patel (2005). Transversely rough slider bearings with squeeze film formed by a magnetic fluid. International Journal of Applied Mechanics and Engineering 10.1,53-76.

Naduvinamani, N. B., B. N. Hanumagowda and SyedaTasneem Fathima (2012). Combined effects of MHD and surface roughness on couple-stress squeeze film lubrication between porous circular stepped plates. Tribology international 56,19-29.

Patel, N. S., D. P. Vakharia, and G. M. Deheri (2012). A study on the performance of a magneticfluid- based hydrodynamic short journal bearing. ISRN mechanical engineering.

Patel, R. M., G. M. Deheri, and P. A. Vadher (2010). Performance of a Magnetic fluid based squeeze film between transversely rough triangular plates. Tribology in industry 32.1, 33-39.

Patel, R. M., G. M. Deheri, and Pragna A. Vadher (2010). Performance of a magnetic fluid-based short bearing. Acta Polytechnica Hungarica 7.3 (2010): 63-78.

Prajapati, B. L. (1995). Magnetic-fluid-based porous squeeze films. Journal of magnetism and magnetic materials, 149.1-2,97-100.

Tønder, K., and H. Christensen (1972). Waviness and roughness in hydrodynamic lubrication. Proceedings of the Institution of Mechanical Engineers,186(1),807-812. 